\title{
Nitrogen incorporation and optical studies of GaAsSbN/GaAs single quantum well heterostructures
}

\author{
Kalyan Nunna, S. Iyer, ${ }^{\text {a) }}$ L. Wu, J. Li, and S. Bharatan \\ North Carolina A\&T State University, Greensboro, North Carolina 27411, USA \\ X. Wei \\ NHMFL Florida State University, Tallahassee, Florida 32310, USA \\ R. T. Senger \\ Department of Physics, Bilkent University, 06800 Ankara, Turkey \\ K. K. Bajaj \\ Department of Physics, Emory University, Atlanta, Georgia 30322, USA
}

(Received 19 March 2007; accepted 20 July 2007; published online 11 September 2007)

\begin{abstract}
In this work, the effects of $\mathrm{N}$ incorporation on the optical properties of $\mathrm{GaAsSbN} / \mathrm{GaAs}$ single quantum wells (SQWs) have been investigated using temperature, excitation, and magnetic dependencies of photoluminescence (PL) characteristics. These layers were grown in an elemental solid source molecular beam epitaxy system with a rf plasma $\mathrm{N}$ source. The $\mathrm{N}$ concentrations in the range of $0.5 \%-2.5 \%$ were investigated in this study. The SQW with $\mathrm{N} \sim 0.5 \%$ exhibits a behavior similar to that in an intermediate regime where the contributions from the localized states in the band gap are dominant. The temperature and excitation dependencies of the PL characteristics indicate that for the $\mathrm{N}$ concentration of $0.9 \%$ and above, the alloy behavior is analogous to that of a regular alloy and the changes in optical properties are only marginal. The conduction band effective mass $\left(m_{\text {eff }}\right)$ values computed from the magnetophotoluminescence spectra using a variational formalism and the band anticrossing model are in good agreement and indicate enhanced values of $m_{\mathrm{eff}}$. However, there is no significant variation in $m_{\mathrm{eff}}$ values of QWs for $\mathrm{N} \geqslant 0.9 \%$. Small redshift of about $30-50 \mathrm{meV}$ for the temperature variations from 10 to $300 \mathrm{~K}$ in conjunction with unusually small blueshift observed in the excitation dependence of PL for $\mathrm{N} \geqslant 0.9 \%$ indicate that this system holds a great promise for laser applications at $1.55 \mu \mathrm{m}$ and beyond. (C) 2007 American Institute of Physics. [DOI: 10.1063/1.2777448]
\end{abstract}

\section{INTRODUCTION}

The dilute nitride alloy quantum wells (QW) lattice matched to GaAs currently under consideration for optical communications in the 1.3 and $1.55 \mu \mathrm{m}$ wavelength regions are $\operatorname{InGaAsN}, \mathrm{GaAsSbN}$, and $\operatorname{InGaAs}(\mathrm{Sb}) \mathrm{N}$ systems. The InGaAsN/GaAs has been established as a successful material system for laser device applications in the $1.3 \mu \mathrm{m}$ region. Extending the wavelength of operation to $1.55 \mu \mathrm{m}$ in this alloy system dictates $\mathrm{N}$ and In concentrations exceeding $2 \%$ and $35 \%$, respectively, resulting in considerable degradation of the structural and optical properties. ${ }^{1-5}$ However, addition of $\mathrm{Sb}$ to this system allowed enhanced In incorporation resulting in redshifting the wavelength further to $1.55 \mu \mathrm{m}$ with improved material quality. ${ }^{1-5}$ Vertical cavity surface emitting lasers (VCSEL) have been successfully demonstrated at $1.45 \mu \mathrm{m}$ using this quinary system. ${ }^{5}$ However, the presence of an additional component makes the system more complex.

$\mathrm{GaAsSbN/GaAs}$ system is another candidate that has shown great promise for application in this wavelength region. Initial research work on these QWs were predominantly carried out by Harmand and co-workers, ${ }^{6-8}$ who demonstrated a band gap reduction of $180 \mathrm{meV}$ in $\mathrm{GaAsSb}$ for a

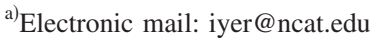

$\mathrm{N}$ concentration of about $1 \%$. Emissions as long as 1.57 and $1.35 \mu \mathrm{m}$ were achieved in annealed $\mathrm{GaAsSb}_{0.15} \mathrm{~N}_{0.025}$ and $\mathrm{GaAsSb}_{0.25} \mathrm{~N}_{0.01} \mathrm{QWs},{ }^{7,8}$ respectively, though the former system exhibited poor luminescence efficiency. The low temperature photoluminescence (PL) characteristics in this system are reported ${ }^{7-12}$ to be dominated by localized excitons due to the $\mathrm{N}$ induced localized states and potential fluctuations. Different annealing techniques have been improvised by various groups ${ }^{1-13}$ for the annihilation of $\mathrm{N}$ related states to improve the PL efficiency. Our recent work ${ }^{9,13}$ indicates that in situ annealing in an As ambient is more effective in the reduction of the density of localized states and nonradiative recombination centers that are responsible for PL thermal quenching. Room temperature (RT) emission wavelength as long as $1.52 \mu \mathrm{m}$ has been achieved ${ }^{14}$ in these in situ annealed QWs at relatively lower composition of $\mathrm{N}$ $(\sim 1.4 \%)$ in comparison to those reported by Harmand et al. ${ }^{7,8}$

The conduction band (CB) effective mass $\left(m_{\mathrm{eff}}\right)$ in this system has been computed by Senger et al. ${ }^{15}$ from the simulation of diamagnetic shift following a variational approach. The effective mass of the QW system with $\mathrm{Sb}$ and $\mathrm{N}$ compositions of $30 \%$ and $1.5 \%$, respectively, was calculated to be $00.9 m_{0}$, almost $50 \%$ higher than in their reference $\mathrm{GaAsSb}$ system. 
In this paper, we have carried out a detailed and systematic study of the effect of $\mathrm{N}$ concentration $(0.5 \%-2.5 \%)$ on the structural and PL characteristics and their dependencies on temperature, incident laser excitation levels, and magnetic field in the in situ annealed GaAsSbN/GaAs single QW (SQW) heterostructures. The values of $m_{\text {eff }}$ have been calculated utilizing the same variational formalism as in Ref. 15, assuming complete localization of holes. These values were compared to those estimated from the band anticrossing (BAC) model. We find that the optoelectronic properties of these QWs are almost invariant with the $\mathrm{N}$ incorporation for $0.9 \% \mathrm{~N}$ and above.

\section{EXPERIMENTAL DETAILS}

The GaAsSbN/GaAs SQW structures were grown on undoped (100) GaAs substrates by solid source molecular beam epitaxy (MBE) with elemental Ga/Al, As/Sb cracker and an rf generated $\mathrm{N}$ plasma sources. The GaAsSbN QW layer of about $90 \AA$ was sandwiched between GaAs layers followed by GaAlAs to improve electron confinement in the QW. The growth temperatures of the QWs and GaAlAs barriers were 470 and $580{ }^{\circ} \mathrm{C}$, respectively, and these samples were exposed to $\mathrm{Sb}$ and As flux prior to QW growth. These were also subjected to in situ annealing in As ambient at $650{ }^{\circ} \mathrm{C}$ for $10 \mathrm{~min}$ to improve the luminescence. ${ }^{13}$

High resolution $\mathrm{x}$-ray diffraction (HRXRD) was performed with a Bede Scientific Metrix-F automated diffractometer, equipped with a microsource $\mathrm{x}$-ray generator. Secondary ion mass spectroscopy (SIMS) measurements were also carried out using a $\mathrm{Cs}^{+}$primary ion beam with beam energy of $1-3 \mathrm{KeV}$. The compositions of $\mathrm{Sb}(28 \%-30 \%)$ and $\mathrm{N}$ $(0.5 \%-2.5 \%)$ were determined from the best fit simulations of the HRXRD spectra in combination with the SIMS analysis (details in Ref. 13). For reference, a GaAsSb SQW was also grown under similar conditions. The PL measurements were carried out using $\mathrm{He}-\mathrm{Ne}$ laser as a light source for excitation and a $0.32 \mathrm{~m}$ double grating monochromator for wavelength dispersion with a liquid nitrogen cooled InGaAs detector. A closed cycle three stage APD cryogenic system was used to study the variation of the PL characteristics in the 10 to $300 \mathrm{~K}$ temperature range. Laser excitation dependence on these QW structures were performed in the range of 10-326 $\mathrm{mW}$ excitation power.

Low temperature $(4 \mathrm{~K})$ magnetophotoluminescence (MPL) measurements were carried out with the magnetic field $(B)$ varying up to $32 \mathrm{~T}$ and directed normal to the sample. The details of the MPL setup are given in Ref. 15 .

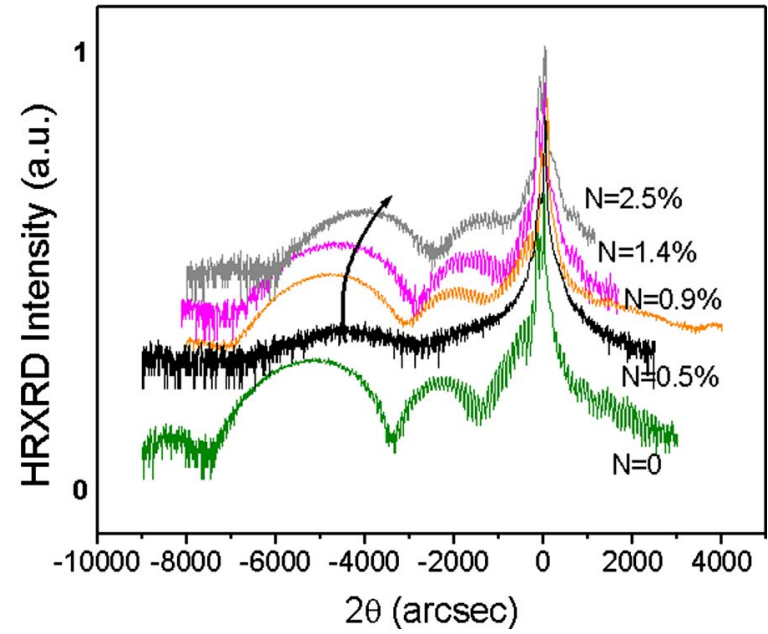

FIG. 1. (Color online) (004) HRXRD $\theta-2 \theta$ scan spectra for GaAsSbN SQWs with increasing $\mathrm{N}$ concentration up to $2.5 \%$.

\section{RESULTS}

Figure 1 illustrates the HRXRD (004) scan spectra of GaAsSbN SQWs for various $\mathrm{N}$ concentrations. With increasing $\mathrm{N}$ composition $(0.5 \%-2.5 \%)$, the QW layer peak shifts closer to the substrate peak as expected. Pendullosung fringes are observed in all the samples.

Figure 2(a) shows the variation of the Sb concentrations in the SQWs with the $\mathrm{N}$ incorporation. No significant change in the $\mathrm{Sb}$ concentration within the experimental error is observed for $\mathrm{N}$ concentrations up to $2.5 \%$. The SIMS depth profile [Fig. 2(b)] indicates that the incorporation of both $\mathrm{N}$ and $\mathrm{Sb}$ in the QW layer begins at the same location, however, the $\mathrm{N}$ profile terminates prior to that of $\mathrm{Sb}$.

The PL peak energy decreases at the rate of $270 \mathrm{meV} / \% \mathrm{~N}$ up to a $\mathrm{N}$ concentration of $\sim 1.4 \%$ and thereafter the rate of reduction diminishes considerably, as displayed in Fig. 3. The PL full width at half maximum (FWHM) increases initially with $\mathrm{N}$ incorporation and remains invariant with further increase.

To get insight into the PL localized states and recombination mechanisms, dependence of the PL peak energy on the temperature and laser excitation power at different $\mathrm{N}$ concentrations was carried out. Figures 4(a) and 4(b) show the PL spectral variations with the temperature for the GaAsSbN SQWs of $\mathrm{N} \sim 0.5 \%$ and $1.4 \%$, respectively. The low temperature PL spectrum corresponding to $\mathrm{N} \sim 0.5 \%$ exhibited a somewhat broad peak that could be resolved into a doublet about $35 \mathrm{meV}$ apart, while in the rest of the samples,
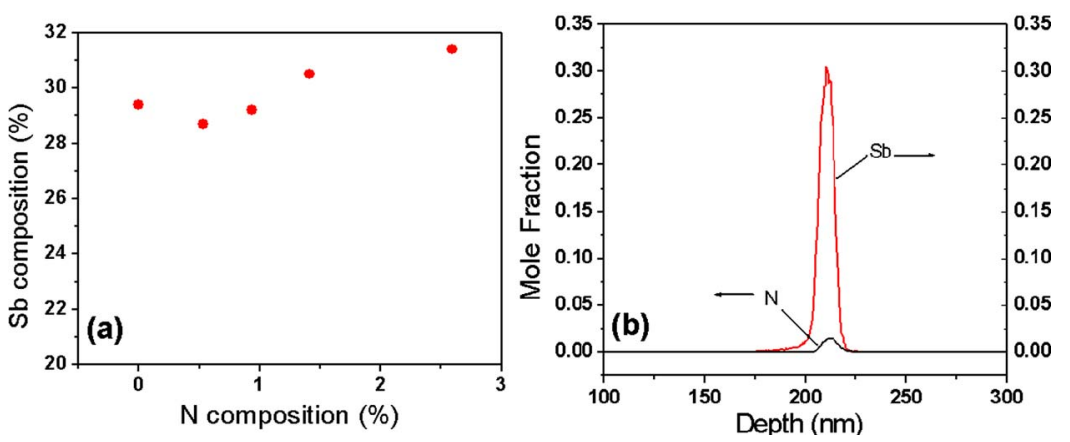

FIG. 2. (Color online) (a) Variation of the Sb compositions in the SQW layers with $\mathrm{N}$ concentration varying up to $2.5 \%$. (b) The SIMS depth profiles of the $\mathrm{Sb}$ and $\mathrm{N}$ incorporations in the GaAsSbN SQW with $\mathrm{Sb}$ $\sim 30 \%$ and $\mathrm{N} \sim 1.4 \%$. 


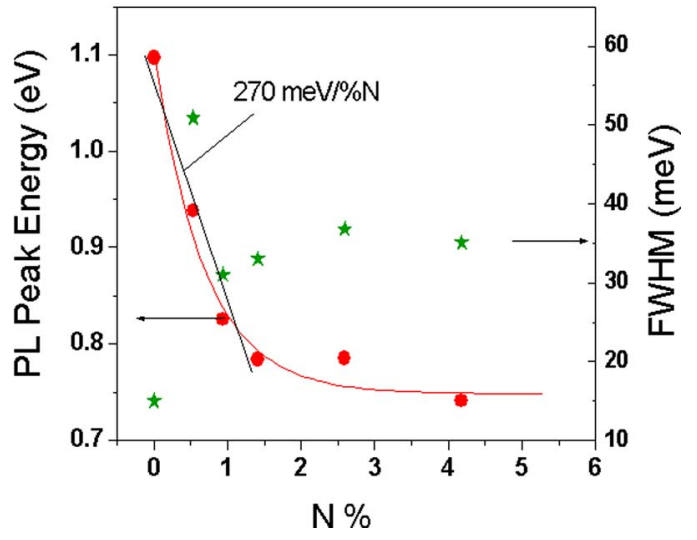

FIG. 3. (Color online) PL peak energy shift and FWHM variation at $10 \mathrm{~K}$ for different $\mathrm{N}$ concentrations.

only a single peak is observed [Fig. 4(b)]. The PL line shape is asymmetric at the lower temperatures exhibiting a long low energy tail, and it becomes more symmetric with increasing $\mathrm{N}$ concentration (though shown only for $\mathrm{N} \sim 1.4 \%$ ), as well as with measurement temperatures beyond $\sim 75 \mathrm{~K}$.

The PL peak energy in the low temperature regime exhibits a (red-blue-red)shift, as shown in Fig. 5(a). This is a characteristic $S$-curve behavior observed up to about $150 \mathrm{~K}$ in the dilute nitride material systems. With further increase in temperature, the PL peak energy decreases monotonically with temperature. The $S$-curve behavior is less pronounced for samples with higher $\mathrm{N}$ concentrations. The fit to the temperature dependence of the PL peak energy is obtained using Varshni's empirical relation. ${ }^{9}$ The fitting parameters, such as $\alpha$ and $\beta$ and the values of the exciton localization parameters, such as $T_{\text {trans }}, E_{\text {loc }}{ }^{\max }, T_{\text {deloc }}$, described in detail in Ref. 9, are listed in Table I and are shown in Fig. 5(a). The values of the latter set of parameters decrease with an increase in the $\mathrm{N}$ concentration.

Figure 5(b) illustrates the corresponding temperature dependence of the total integrated PL intensity $\left(I_{\mathrm{PL}}\right)$ in these SQWs. The intensity of the PL peak drops rapidly over two orders of magnitude with rise in the temperature above $\sim 50 \mathrm{~K}$. Excellent fit to the temperature dependence of the PL peak intensity data is obtained using a phenomenological expression given in Ref. 9, which assumes the presence of two nonradiative recombination channels. The activation energies of the two different nonradiative channels $E_{a}$ and $E_{b}$ and the ratio of the relative efficiency of these two nonradiative recombination mechanisms, $A / B$, for the different $\mathrm{N}$ concentrations are given in Table I.
Figures 6(a) and 6(b) demonstrate the PL spectra at $10 \mathrm{~K}$ as a function of the laser power for the $\mathrm{N}$ concentrations of $0.9 \%$ and $2.5 \%$, respectively. At low excitation levels, the PL line shape is asymmetric, with an exponential low energy tail being more pronounced for the $\mathrm{QW}$ of the lowest $\mathrm{N}$ concentration. The PL line shape becomes more symmetric with the increasing excitation power. For $\mathrm{N} \geqslant 1.4 \%$, a shoulder evolves on the higher energy side of the emission peak [shown in Fig. 6(b) for $2.5 \% \mathrm{~N}$ ]. A blueshift of $\sim 11 \mathrm{meV}$ is observed in the reference GaAsSb and drops to $0.4 \mathrm{meV}$ for $\mathrm{N} \geqslant 1.4 \%$ with increase in the excitation power from 10 to $326 \mathrm{~mW}$, as shown in Fig. 7.

Figures 8(a) and 8(b) show the dependencies of the integrated PL intensity and FWHM on incident laser power at different $\mathrm{N}$ concentrations. The integrated PL intensity is fitted using the power law of the form $I_{\mathrm{PL}}=a P^{b}$, where $I_{\mathrm{PL}}$ and $P$ are the integrated PL and laser intensities, respectively. The fitting parameters $a$ and $b$ are representative of the overall PL efficiency and the nature of the recombination mechanisms, respectively. The FWHM of PL spectra of the GaAsSb QW increases with the laser power, while for $\mathrm{N}$ $\sim 0.5 \%$, it exhibits an opposite trend. For all the other $\mathrm{N}$ concentrations, the FWHM is found to be invariant with laser power.

Figure 9 shows the variation of the diamagnetic shift $\delta$ defined as $E_{g}(B)-E_{g}(B=0)$, as a function of applied magnetic field for different $\mathrm{N}(0 \%-2.5 \%)$ concentrations. The diamagnetic shift of the nitride QWs do not vary significantly with the $\mathrm{N}$ concentration and is less than half of that observed in the GaAsSb QW. The best fit simulations of these shifts have been performed using a variational approach with the $\mathrm{CB}$ electron mass as a fitting parameter and assuming the heavy-hole mass to be infinite, as in Ref. 15 . The $m_{\text {eff }}$ thus computed for the GaAsSb SQW is $0.05 m_{0}$, which is enhanced to $0.070 m_{0}-0.088 m_{0}$ for the nitrides depending on the $\mathrm{N}$ composition.

The above values are compared with those computed using the BAC model. Assuming the low temperature PL peak energy to be the band gap of the QWs, the experimental PL peak energy data for different $\mathrm{N}$ concentrations has been fitted using the following energy dispersion relation from the BAC model: ${ }^{16-18}$

$$
E_{\mathrm{gp}}=\frac{E_{M}(k)+E_{N}-\sqrt{\left(E_{M}(k)-E_{N}\right)^{2}+4 V^{2} x}}{2},
$$

where $E_{N}$ is the resonant energy level introduced by $\mathrm{N}$, $E_{M}(k)$ is the interacting CB energy level of the host semicon-
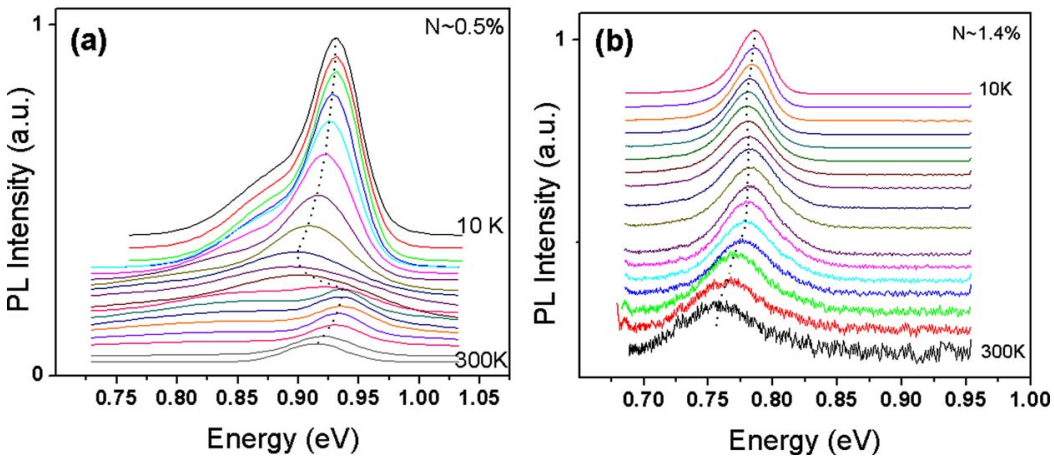

FIG. 4. (Color online) PL lineshape dependence on the temperature in (a) $\mathrm{N} \sim 0.5 \%$ and (b) $\mathrm{N} \sim 1.4 \%$ SQWs. 

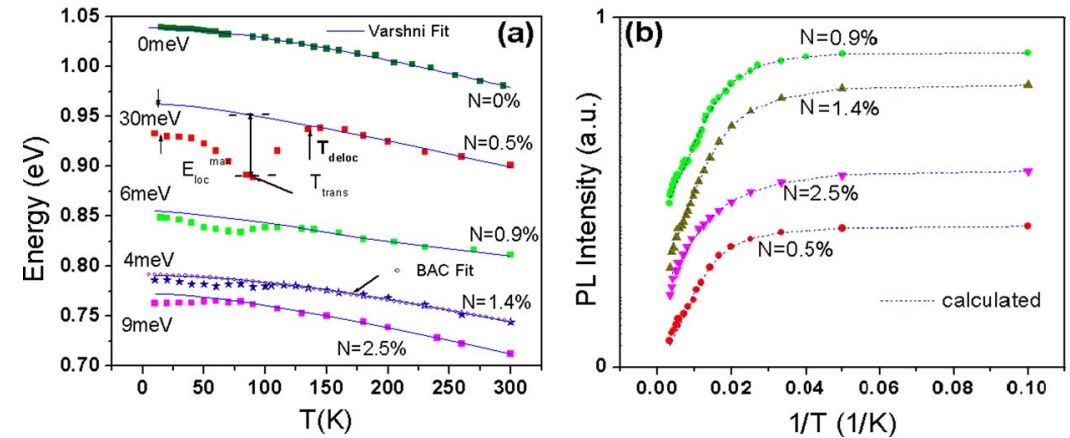

FIG. 5. (Color online) Temperature dependence: (a) PL peak energy and (b) integrated PL peak intensity of the in situ annealed GaAsSbN/GaAs SQW heterostructures for different $\mathrm{N}$ concentrations. The experimental data curve of $\mathrm{N} \sim 2.5 \%$ in (a) has been offset by $25 \mathrm{meV}$ vertically for clarity. The superimposed solid curves are fits to the Varshni's relation (Ref. 9) and the open circles are the fits using the BAC model. The dashed curves in (b) are the fitting curves (fitting equation from Ref. 9). ductor GaAsSb, $V$ represents the coupling parameter between the $\mathrm{N}$ states and the extended states, $x$ is the nitrogen mole fraction. $E_{N}=1.65 \mathrm{eV}$ above the valence band edge, and $V$ is typically in the range of $2.4-2.7 \mathrm{eV}$ for GaAsN and InGaAsN material systems. ${ }^{16-18}$ The $\mathrm{N}$ energy level is assumed to be independent of the temperature as well as in the variation in the $\mathrm{Sb}$ composition. The BAC parameters of $E_{N}$ and $V$ of 1.65 and $3.9 \mathrm{eV}$, respectively, are found to provide a best fit to our PL peak energy variation with N\%. These parameters were further confirmed by fitting the temperature dependence of the PL peak energy of the nitride QWs, where the temperature dependence of the host energy level was taken into account using our empirical values of $\alpha$ and $\beta$, as described earlier. Fitting with the BAC model is shown in Fig. 5(a) for one sample with $\mathrm{N} \sim 1.4 \%$ only for clarity. It has to be noted that the BAC fitting did not include the QW with $\mathrm{N} \sim 2.5 \%$, as there is little band gap reduction with $\mathrm{N} \%$ beyond $\mathrm{N} \sim 1.4 \%$. The $m_{\text {eff }}$ values are then estimated from the following analytical expression derived from the dispersion relation

$$
m_{\mathrm{eff}}=m^{*}\left[1+\left(\frac{V x^{1 / 2}}{E_{N}-E_{M}}\right)^{2}\right],
$$

where $m^{*}$, the effective mass of the electron in the host $\mathrm{GaAsSb}$, is taken to be $0.05 m_{0}$ determined from the MPL data discussed earlier. The $m_{\text {eff }}$ values thus calculated from the above approaches are compared in Fig. 10.

\section{DISCUSSION}

The presence of the Pendullosung fringes in the $\mathrm{x}$-ray rocking curves of all the nitride QWs indicates sharp interfaces and excellent crystalline quality of the grown layers (Fig. 1). With the increasing $\mathrm{N}$ concentration, the QW layer becomes more tensile strained as is evident from the shift of the QW peak towards the GaAs substrate peak. Sb composi- tion was found to be relatively independent of the $\mathrm{N}$ incorporation, consistent with the reports in the literature. ${ }^{5}$

The SIMS depth profiles in Fig. 2(b) indicates sharp buffer/QW interface in comparison to QW/cap layer. The $\mathrm{Sb}$ profile is broader than the $\mathrm{N}$ profile due to the trailing edge of $\mathrm{Sb}$ even after closing the $\mathrm{Sb}$ source shutter caused by the surfactant nature of Sb. Harris, ${ }^{5}$ on the contrary, found that both the $\mathrm{N}$ and $\mathrm{Sb}$ profiles do not begin as well as end at the same location in their GaAsSbN QW sample $(\mathrm{N} \sim 3 \%$ and $\mathrm{Sb} \sim 10 \%)$. Hence, exposing the sample to As and $\mathrm{Sb}$ fluxes prior to the QW growth seems to improve the buffer/QW interface. This is also consistent with the results obtained using cross sectional transmission electron microscopy study of our samples, where the interface roughness was found to be smaller on the buffer/epilayer interface $(0.3 \mathrm{~nm})$ in comparison to the epilayer/cap interface $(0.8 \mathrm{~nm}) .^{19}$

The PL peak energy decreases at a rate of $270 \mathrm{meV} / \% \mathrm{~N}$ for the $\mathrm{N}$ concentration varying up to $1.4 \%$; thereafter, the variation is minimal, as shown in Fig. 3. The initial rapid band gap reduction with increasing $\mathrm{N}$ concentration in these QWs is commonly attributed ${ }^{20}$ to both the lowering of the conduction band as well as increase in the conduction band effective mass. The latter tends to saturate for $\mathrm{N} \geqslant 0.9 \%$ as discussed later, which partly accounts for the observed small rate of reduction in the PL peak energy. We have achieved a band gap reduction of $\sim 328 \mathrm{meV}$ for $\mathrm{N} \sim 1.4 \%$ and $\mathrm{Sb}$ $\sim 30 \%$ with respect to the GaAsSb $(30 \% \mathrm{Sb}),{ }^{21}$ while other groups observed a reduction of $\sim 230 \mathrm{meV}$ for $\mathrm{N} \sim 1.2 \%$ and $\mathrm{Sb} \sim 26 \%,^{6} \sim 196 \mathrm{meV}$ for $\mathrm{N} \sim 1.5 \%$ and $\mathrm{Sb} \sim 30 \%,{ }^{15}$ $\sim 155 \mathrm{meV}$ for $\mathrm{N} \sim 1 \%,{ }^{3}$ and $\sim 85 \mathrm{meV}$ for $\mathrm{N} \sim 0.7 \%$ and $\mathrm{Sb} \sim 15 \%,{ }^{8}$ with respect to their reference GaAsSb QW. Further, the energy reduction in our work is also relatively higher than those observed in the other dilute nitride systems, namely, GaAsN: $290 \mathrm{meV}$ for $\sim 1.6 \% \mathrm{~N},{ }^{6} 250 \mathrm{meV}$ for $\mathrm{N} \sim 1 \%{ }^{18}$ from the host GaAs, ${ }^{18}$ InGaAsN: $\sim 314 \mathrm{meV}$ for $\mathrm{N} \sim 2.5 \%,{ }^{2} \sim 180 \mathrm{meV}$ for $\sim 1.7 \%$ of $\mathrm{N}$ with respect to

TABLE I. Varshni's parameters for the temperature dependence of the PL peak position and total integrated PL peak intensity for different $\mathrm{N}$ concentrations.

\begin{tabular}{lcccccccccc}
\hline \hline $\mathrm{N} \%$ & $E_{10 \mathrm{~K}}$ & $\begin{array}{c}\alpha \\
(\mathrm{meV} / \mathrm{K})\end{array}$ & $\begin{array}{c}\beta \\
(\mathrm{K})\end{array}$ & $\begin{array}{c}E_{\mathrm{loc}}(10 \mathrm{~K}) \\
(\mathrm{meV})\end{array}$ & $\begin{array}{c}E_{\mathrm{loc}}{ }^{\max } \\
(\mathrm{meV})\end{array}$ & $\begin{array}{c}T_{\text {trans }} \\
(\mathrm{K})\end{array}$ & $\begin{array}{c}T_{\text {deloc }} \\
(\mathrm{K})\end{array}$ & $\begin{array}{c}E_{a} \\
(\mathrm{meV})\end{array}$ & $\begin{array}{c}E_{b} \\
(\mathrm{meV})\end{array}$ & $A / B$ \\
\hline 0 & 1.039 & 0.36 & 245 & $\ldots$ & $\ldots$ & $\ldots$ & $\ldots$ & 24 & 5.1 & 23 \\
0.5 & 0.932 & 0.28 & 100 & 30 & 62 & 90 & 165 & 26 & 7 & 37 \\
0.9 & 0.823 & 0.37 & 273 & 6 & 16 & 75 & 100 & 22 & 5.2 & 42 \\
1.4 & 0.786 & 0.36 & 325 & 4 & 8 & 60 & 90 & 26 & 7.6 & 42 \\
2.5 & 0.788 & 0.33 & 204 & 9 & 9 & 10 & 80 & 22 & 4.9 & 10 \\
\hline \hline
\end{tabular}



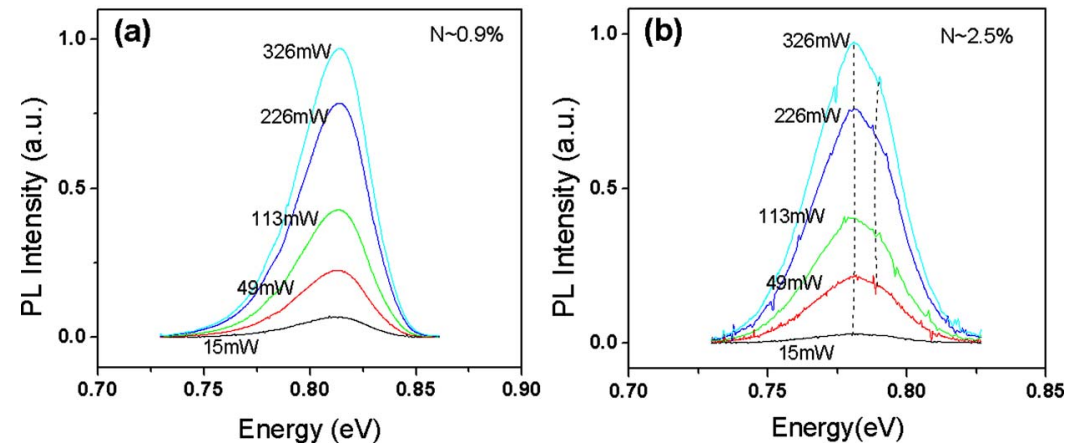

FIG. 6. (Color online) PL lineshape dependence on the laser excitation power at $10 \mathrm{~K}$ for (a) $\mathrm{N} \sim 0.9 \%$ and (b) $\mathrm{N} \sim 2.5 \%$.

InGaAs, ${ }^{22}$ GaInNAsSb: $\sim 50 \mathrm{meV}$ for $\mathrm{N} \sim 0.6 \%$ with reference to GaInNAs, ${ }^{3}$ and $\sim 320 \mathrm{meV}$ for $\sim 1.5 \% \mathrm{~N}$ in $\mathrm{GaSbN}$ from the host $\mathrm{GaSb}^{23}$ This stated range of band gap reduction due to $\mathrm{N}$ is made based on similar compositions in both the non-nitride and nitride materials to get a better estimate of reduction due to $\mathrm{N}$ incorporation. Thus, the reduction in the band gap energy, with respect to the corresponding host non-nitride alloys, ranges from 105 to $250 \mathrm{meV} / \% \mathrm{~N}$ in various dilute nitride III-V systems. ${ }^{2,3,6,8,15,18,22,23}$ It may be noted that, in comparison to other III-As based $\mathrm{N}$ alloys, the redshift in the emission wavelength is greater in $\mathrm{Sb}$ or mixed $\mathrm{Sb}-\mathrm{As}$ based system. A similar conclusion has been drawn by Harmand et al. ${ }^{24}$ where they report the reductions of 127 , 124 , and $151 \mathrm{meV} \% \mathrm{~N}$ contributions due only to $\mathrm{N}$ in the annealed GaAsN, InGaAsN, and GaAsSbN systems, respectively, relative to GaAs after carefully excluding the strain effects.

A $1.7 \mu \mathrm{m}$ RT emission with a FWHM of $67 \mathrm{meV}$ has been achieved for a SQW with $\mathrm{N} \sim 1.4 \%$ and $\mathrm{Sb} \sim 30 \%{ }^{21}$ RT emission of $1.53 \mu \mathrm{m}$ with a FWHM of $45 \mathrm{meV}$ has also been achieved on another in situ annealed SQW sample. These values are comparable to the best reported RT emission "near" $1.5 \mu \mathrm{m}$ of $35 \mathrm{meV}$ at $1.48 \mu \mathrm{m}$ (Refs. 3 and 24) but somewhat higher than $28.7 \mathrm{meV}$ at $1.45 \mu \mathrm{m}$ (Ref. 4) after annealing in GaInNAsSb system.

The $S$-curve behaviors observed at low temperatures in the temperature dependence of the PL peak energy spectra of the GaAsSbN SQWs, as shown in Fig. 5(a), are commonly attributed to the localized behavior of the excitons, due to the $\mathrm{N}$ induced potential fluctuations. This behavior has been discussed in detail in our earlier work. ${ }^{9}$ The maximum localization energy is unusually large $(\sim 62 \mathrm{meV})$ for $0.5 \% \mathrm{~N}$ sample and decreases drastically for higher $\mathrm{N} \%$. The localization energy defined as $E_{\mathrm{loc}}(T)=E_{g}(T)-E_{\mathrm{PL}}(T)$ at $T=10 \mathrm{~K}$ is also large $(30 \mathrm{meV})$ for $\mathrm{N} \sim 0.5 \%$, and, thereafter, it drops to 4-9 meV at higher $\mathrm{N} \%$. Likewise, $T_{\text {trans }}$ and $T_{\text {deloc }}$ exhibit a decrease with increase in N\%. The behavior of the PL spectrum of $\mathrm{N} \sim 0.5 \%$ can be explained if the $\mathrm{N}$ concentration in this sample is considered to be in the intermediate dilute/ amalgamated region between the impurity and the regular alloy. In this intermediate regime, the PL spectrum consists of excitonic emissions bound to localized states below the band edge as well as from spatially distributed potential wells resulting in a broad spectrum. With a slight increase in temperature, the excitons bound to the localized potentials relax into deeper potential wells and localized states, resulting in a redshift of the PL peak energy. With further increase in the temperature above $T_{\text {trans }}$, the excitons are delocalized and the transitions from the conduction band start to take over, following Varshni's relation. This high $E_{\text {loc }}^{\max }$ was also witnessed in another sample with similar low $\mathrm{N}$ concentration, which exhibited additional secondary peaks (not shown here). Hence, we do not believe it as a sample/growth based artifact. This is consistent with the observations in other nitride systems, such as GaAsN, where this transition region is reported to occur in the range of $0.4 \%-1.0 \% .^{25}$ The PL lineshape is asymmetric for this sample with low energy tail longer, in comparison to the other nitride samples, which further attests to the assignment of dilute/amalgamated region at this $\mathrm{N}$ concentration.

For $\mathrm{N} \geqslant 0.9 \%$ the values of $E_{\text {loc }}(10 \mathrm{~K})$ are comparable to those reported in the InGaAsN QWs (Refs. 10, 11, 26, and 27) but somewhat higher than those observed in the InGaAsNSb QW (Ref. 4) for similar N concentrations. However, these comparisons have to be made with great caution, as the localization energy depends on the laser excitation intensity in addition to the material quality and $\mathrm{N} \%$. The decrease in $E_{\text {loc }}(10 \mathrm{~K})$ with $\mathrm{N} \%$ is contrary to the observations of InGaAsN/GaAs QWs where it is found to increase with $\mathrm{N} \%{ }^{26}$ The low values of $E_{\mathrm{loc}}{ }^{\max }$ and $E_{\mathrm{loc}}(10 \mathrm{~K})$ for $\mathrm{N} \geqslant 0.9 \%$ are clear evidence of shallow potential fluctuations in these samples. More symmetric PL line shape and the absence of a low energy tail in these samples further attest to the above inference.

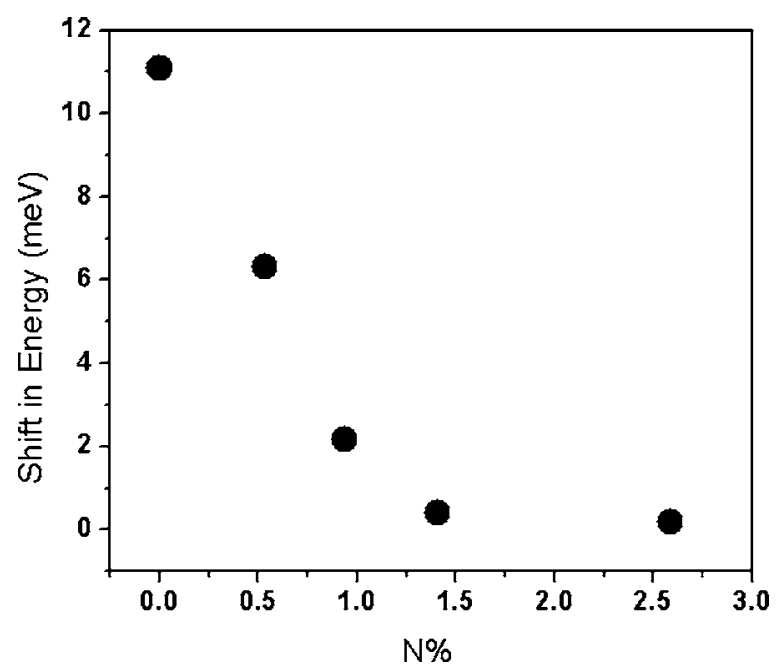

FIG. 7. Low temperature PL peak energy shift with the laser excitation power increase from 10 to $326 \mathrm{~mW}$ for different $\mathrm{N}$ concentrations. 

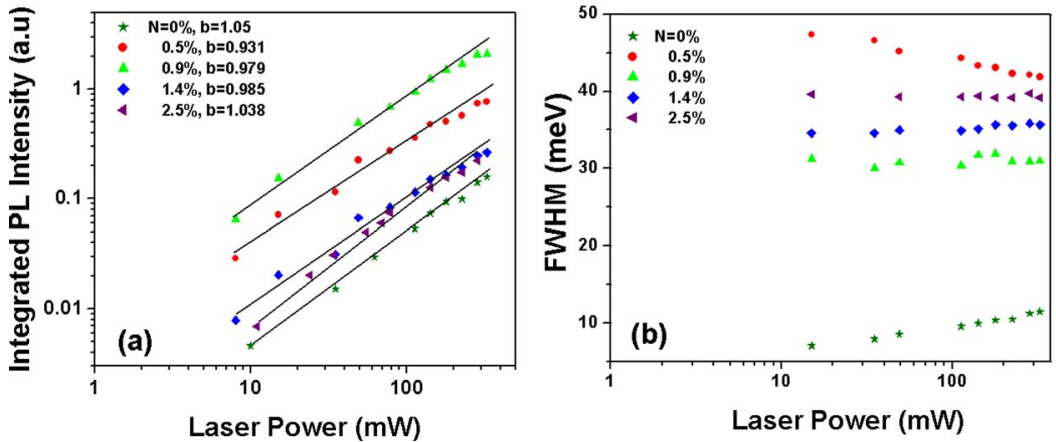

FIG. 8. (Color online) Integrated PL intensity data as a function of the laser excitation power for different $\mathrm{N}$ concentrations. The superimposed solid lines are the fits to the equation $I_{\mathrm{PL}}(P)=a P^{b}$ to the experimental data. (b) The corresponding variation of the FWHM with excitation power for different $\mathrm{N}$ concentrations.
The above conjectures about the $\mathrm{N}$ dependence are also borne out in the excitation dependence of the FWHM and the blueshift observed in the PL peak energy. The linewidth of the PL emission exhibits an increase with laser excitation in the GaAsSb QW, as expected due to level filling in the traditional alloys [Fig. 8(b)]. On the other hand, in the case of $\mathrm{N} \sim 0.5 \%$, the PL linewidth shows a slight decrease with increasing laser power. This further supports the highly localized nature of the states in this sample, as increase in the laser power would contribute to the population of bound excitons resulting in a narrower linewidth. At higher N\%, no significant variation in the FWHM with a change in excitation level is observed. Further, the PL spectra at these high N concentrations can be resolved into two closely spaced peaks, separated by $\sim 9 \mathrm{meV}$, similar to those observed by Saidi et $a l .{ }^{28}$ in GaAsN epilayers. The absence of this additional peak in the QWs with low $\mathrm{N}$ concentration suggests that this may be related to a modulation of the band structure.

The values of $b$ obtained from fitting the laser power dependence of the integrated PL intensity data are $\sim 1$, suggesting the dominance of free exciton recombination [Fig. 8(a)]. The values of the activation energies $E_{a}$ and $E_{b}$ for the nonradiative recombination channels, as determined from the temperature dependence of the integrated PL intensity are all similar.

The blueshift of the PL peak decreases with the N\% for

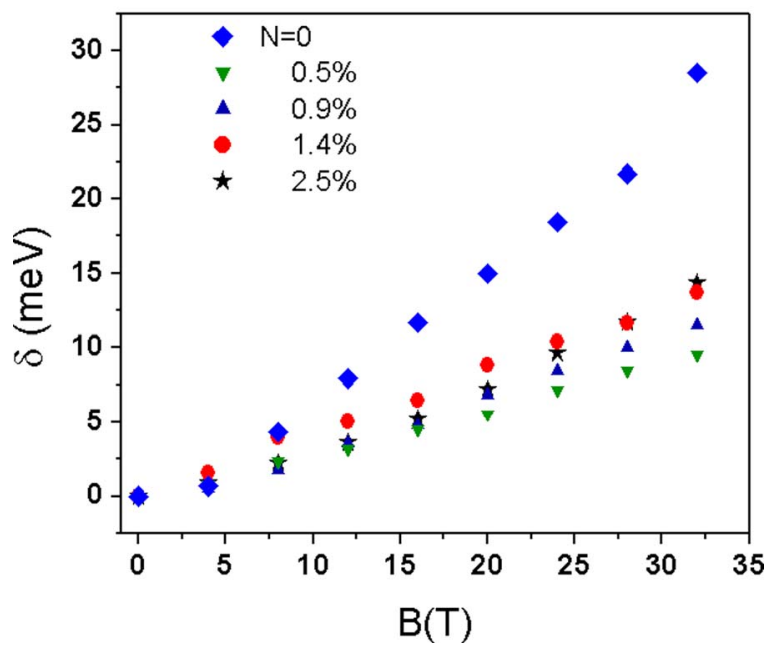

FIG. 9. (Color online) The variation of the diamagnetic shifts as a function of magnetic field for the ternary and the quaternary SQWs grown at different $\mathrm{N}$ concentrations. the excitation power increase from 10 to $326 \mathrm{~mW}$ (Fig. 7) and is $\sim 0.4 \mathrm{meV}$ for $\mathrm{N} \geqslant 1.4 \%$. This shift is much smaller than the values of $18-38 \mathrm{meV}$ observed in GaAsN QWs (Refs. 29 and 30) and in InGaAsN QWs (Ref. 27) (extrapolated from the graphs in the mentioned references) for almost two orders of magnitude increase in excitation power. This again reflects the lesser potential fluctuations in our samples.

This is also consistent with the smaller shift observed in the temperature dependence data. It is worth noting that the overall redshift from 10 to $300 \mathrm{~K}$ is in the range of $30-50 \mathrm{meV}$ for $\mathrm{N} \geqslant 0.9 \%$, considerably smaller than those reported in the literature on the GaAsSbN/GaAs QWs and epilayers ${ }^{7,8,12,31}$ and comparable to some of the lowest values reported for GaAsN (Refs. 28 and 32) and InGaAsN (Refs. 11 and 26) QWs and epilayers.

We have analyzed the variation of the low temperature PL transition energy with magnetic field $(0-32 \mathrm{~T})$. The diamagnetic shift of this excitonic transition is calculated using the variational formalism as described in Ref. 15. In comparison to the GaAsSb QW, the effect of incorporating small amounts of $\mathrm{N}$ is assumed to change only the electron effective mass and the $\mathrm{CB}$ offset value. ${ }^{8}$ We have modeled the low temperature localization of the excitons in these QWs by assuming the heavy-hole mass to be infinite, and by treating the electron mass as an adjustable parameter. The value of $0.05 m_{0}$ obtained for the GaAsSb QW is somewhat lower than the reported value, ${ }^{15}$ which indicates that the localiza-

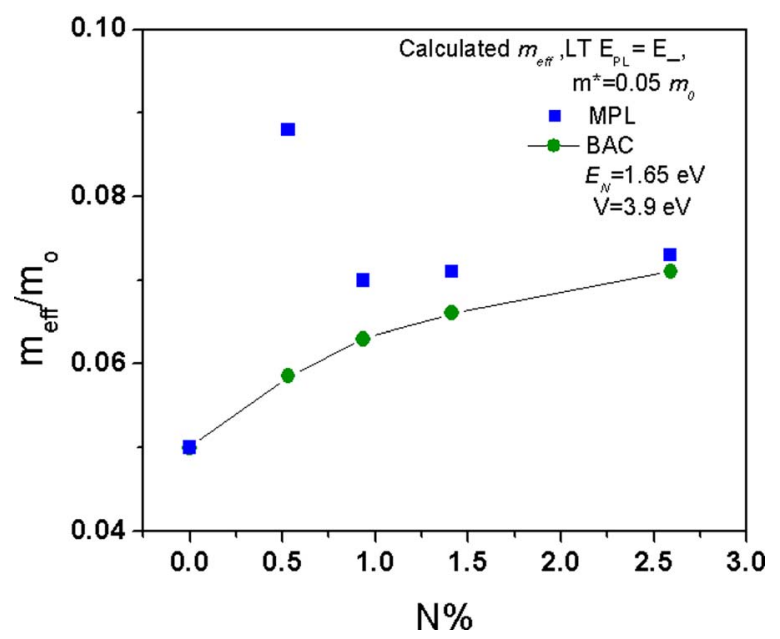

FIG. 10. (Color online) The calculated values of $m_{\text {eff }}$ as a function of $\mathrm{N}$ concentration from the diamagnetic shifts using the variational approach are compared with the corresponding values obtained from the BAC model. 
tion of the excitons in our GaAsSb sample is weaker. Comparatively lower diamagnetic shifts observed in the nitride QWs indicate an enhanced value of the electron effective mass in the GaAsSbN SQWs. The relatively larger value of $m_{\text {eff }}$ calculated for the sample with $0.5 \% \mathrm{~N}$ can be understood in view of the distinctly higher localization energy observed in Fig. 5(a).

The calculated $\mathrm{CB}$ effective mass $\left(0.071 \mathrm{~m}_{0}\right)$ in the GaAsSbN QW with $1.4 \% \mathrm{~N}$ is also slightly lower than that reported by Senger et al. $\left(0.090 m_{0}\right)$ (Ref. 15) in a similar QW with $1.5 \% \mathrm{~N}$. In both the studies, the localization of excitons has been modeled with the assumption of perfectly localized holes, even though the actual physical origin of excitonic localization in these material systems is not completely known. The type and degree of the localization can be related to the growth-dependent defect and the potential fluctuation profiles of a particular sample. It is to be noted that, from the diamagnetic shifts, one can only deduce that the exciton reduced mass information and the quantitative values of the $m_{\text {eff }}$ crucially rely on the assumed value of the hole effective mass. Perfect localization of the hole model provides a lower bound to the $m_{\text {eff }}$ value. In the absence of any reliable values of the localized hole masses in these alloy systems, this model at present provides the best estimate. However, the predicted relative enhancement of $m_{\text {eff }}$ in the nitride QWs (in the range of $40 \%-75 \%$ as a function of $\mathrm{N}$ concentration) should be far less sensitive to the choice of the heavy-hole mass value.

The $m_{\text {eff }}$ values computed from the MPL data are found to be in good agreement with those determined from the BAC model. The value of $E_{N}=1.65 \mathrm{eV}$ that resulted in the best fit to the experimental data is in excellent agreement with the reported values in GaAsN and InGaAsN systems. ${ }^{16-18}$ However, the best fit value of the coupling constant $V=3.9 \mathrm{eV}$ is considerably higher than the reported range of values of $2.4-2.7 \mathrm{eV}$ in the GaAsN and InGaAsN systems. ${ }^{16-18}$ The $\mathrm{N}$ level at $\sim 1.22 \mathrm{eV}$ was also considered based on the valence band edge in the GaAsSb $(\mathrm{Sb} \sim 30 \%)$ (Ref. 33) resulting in lower coupling constant of $2.8 \mathrm{eV}$, giving equally best fit to the data for the PL peak energy variation with the $\mathrm{N} \%$ and temperature. Though the value of $V$ is in excellent agreement with the reported data, the effective mass values calculated using these BAC parameters are found to be much higher than those computed from the MPL data. Thus, $E_{N}=1.65 \mathrm{eV}$ and $V=3.9 \mathrm{eV}$ are considered to be the BAC parameters that best describe the PL energy variation with the $\mathrm{N} \%$ and temperature in the GaAsSbN/GaAs SQWs. However, the high value of $V=3.9 \mathrm{eV}$ cannot be explained. It is well recognized that the BAC model does not take into account many of the effects, namely, interaction of the valence band as well as the density of states of the band tails on $E_{N}$ which may be important for the GaAsSbN/GaAs system.

The above $m_{\text {eff }}$ values determined from the BAC model are found to be in good agreement with those computed from the MPL. However, in both the cases, there is not a significant variation among the $m_{\text {eff }}$ values of different nitride samples, particularly for $\mathrm{N} \geqslant 0.9 \%$, consistent with all other PL characteristics.

\section{v. CONCLUSIONS}

In conclusion, the band gap reduction, effective mass, exciton localization energy at low temperatures, and the PL characteristics show only marginal changes in the in situ annealed GaAsSbN SQWs for $\mathrm{N} \geqslant 0.9 \%$. The $\mathrm{N}$ dependent studies of the PL characteristics such as the peak energy, FWHM, and intensity with temperature and incident laser excitation indicate that $0.5 \% \mathrm{~N}$ corresponds to the dilute/ amalgamated region while $\mathrm{N} \geqslant 0.9 \%$ corresponds to that of a regular alloy. The $\mathrm{CB}$ effective mass values computed from the variational formalism as well as the BAC model are in good agreement. The temperature induced band gap redshift from 10 to $300 \mathrm{~K}$ is only $30-50 \mathrm{meV}$, and the blueshift observed for the laser excitation increase by a factor of $32 \%$ is only $0.4 \mathrm{meV}$. These low values of shifts observed in this system make it a very attractive system for laser devices. Thus, this work indicates that this material system holds great promise for both extending the wavelength further and also for VCSEL devices operating in the $1.55 \mu \mathrm{m}$ region.

\section{ACKNOWLEDGMENTS}

This work was supported by the Army Research Office (Grant No. W911NF-04-1-0025). A partial funding for a student from The Penn State Electro Optics Center (Subcontract No. 0014-4-01150) is also acknowledged. One of the authors (R.T.S.) acknowledges the financial support from TÜBAGEBIP on the effective mass computations. The high resolution x-ray diffraction measurements were confirmed by Dr. Kevin Matney from Bede Scientific Inc, Englewood, Colorado. SIMS measurements were carried out at Evans Analytical Group, New Jersey. The laser excitation and magneto-PL measurements were carried out at the National High Magnetic Field Laboratory, Tallahassee, Florida.

${ }^{1}$ W. Ha, V. Gambin, S. Bank, H. Bae, M. Wistey, H. Yuen, S. Kim, and J. S. Harris, Jr., IEEE J. Quantum Electron. 38, 1260 (2002).

${ }^{2}$ K. Volz, V. Gambin, W. Ha, M. A. Wistey, H. Yuen, S. Bank, and J. S. Harris, J. Cryst. Growth 251, 360 (2003).

${ }^{3}$ L. H. Li, V. Sallet, G. Patriarche, L. Largeau, S. Bouchoule, L. Travers, and J. C. Harmand, Appl. Phys. Lett. 83, 1298 (2003).

${ }^{4}$ S. Bank, M. Wistey, L. Goddard, H. Yuen, V. Lordi, and J. S. Harris, Jr., IEEE J. Quantum Electron. 40, 6 (2004).

${ }^{5}$ J. S. Harris, Jr., J. Cryst. Growth 278, 3 (2005).

${ }^{6}$ G. Ungaro, G. Le Roux, R. Teissier, and J. C. Harmand, Electron. Lett. 35, 1246 (1999)

${ }^{7}$ J. C. Harmand et al., J. Cryst. Growth 553, 227 (2001)

${ }^{8}$ J. C. Harmand et al., Semicond. Sci. Technol. 17, 778 (2002).

${ }^{9} \mathrm{~J}$. Li, S. Iyer, S. Bharatan, L. Wu, K. Nunna, W. Collis, K. Bajaj, and K. Matney, J. Appl. Phys. 98, 013703 (2005).

${ }^{10}$ A. Pinault and E. Tournie, Solid-State Electron. 47, 477 (2003).

${ }^{11}$ A. Pinault and E. Tournie, Appl. Phys. Lett. 78, 1562 (2001).

${ }^{12}$ S. A. Lourenco, I. F. L. Dias, L. C. Pocas, and J. L. Duarte, J. Appl. Phys. 93, 4475 (2003)

${ }^{13}$ L. Wu, S. Iyer, K. Nunna, J. Li, S. Bharatan, W. Collis, and K. Matney, J. Cryst. Growth 279, 293 (2005).

${ }^{14}$ L. Wu, S. Iyer, K. Nunna, S. Bharatan, J. Li, and W. J. Collis, Mater. Res. Soc. Symp. Proc. 799, Z1.9.1-9.7 (2004).

${ }^{15}$ R. T. Senger et al., Appl. Phys. Lett. 83, 5425 (2003).

${ }^{16}$ C. Skierbiszewski et al., Appl. Phys. Lett. 76, 2409 (2000).

${ }^{17}$ J. Wu, W. Shan, W. Walukiewicz, K. M. Yu, J. W. Ager III, E. E. Haller, H. P. Xin, and C. W. Tu, Phys. Rev. B 64, 085320 (2001).

${ }^{18}$ F. Bousbih, S. Ben Bouzid, R. Chtourou, F. F. Charfi, J. C. Harmand, and G. Ungaro, Mater. Sci. Eng., C C21, 251 (2002). 
${ }^{19}$ T. Biggerstaff, G. Duscher, S. J. Pennycook, N. G. Stoddard, and S. Iyer, Philos. Mag. (submitted).

${ }^{20}$ R. Kudrawiec, G. Sek, K. Ryczko, J. Misiewicz, P. Sundgren, C. Asplund, and M. Hammar, Solid State Commun. 127, 613 (2003).

${ }^{21}$ K. Nunna, S. Iyer, L. Wu, S. Bharatan, J. Li, K. K. Bajaj, X. Wei, and R. T. Senger, J. Vac. Sci. Technol. B 25, 1113 (2007).

${ }^{22}$ H. Saito, T. Makimoto, and N. Kobayashi, J. Cryst. Growth 195, 416 (1998).

${ }^{23}$ T. D. Veal et al., Appl. Phys. Lett. 87, 132101 (2005).

${ }^{24}$ J. C. Harmand et al., Dilute Nitride Semiconductors, edited by M. Henin (Elsevier, San Diego, CA, 2005), Chap. 15, pp. 471-505.

${ }^{25}$ B. S. Ma, F. H. Su, K. Ding, G. H. Li, Y. Zhang, A. Mascarenhas, H. P. Xin, and C. W. Tu, Phys. Rev. B 71, 045213 (2005).

${ }^{26}$ J. Misiewicz, P. Sitarek, K. Ryczko, R. Kudrawiec, M. Fischer, M. Reinhardt, and A. Forchel, Microelectron. J. 34, 737 (2003).
${ }^{27}$ E. M. Pavelescu, T. Jouhti, C. S. Peng, W. Li, J. Konttinen, M. Dumitrescu, P. Laukkanen, and M. Pessa, J. Cryst. Growth 241, 31 (2002).

${ }^{28}$ F. Saidi, F. Hassen, H. Maaref, L. Auvray, H. Dumont, and Y. Monteil, Mater. Sci. Eng., C C21, 245 (2002).

${ }^{29}$ I. A. Buyanova, W. M. Chen, G. Pozina, J. P. Bergman, B. Monemar, H. P. Xin, and C. W. Tu, Appl. Phys. Lett. 75, 501 (1999).

${ }^{30}$ H. Dumont, L. Auvray, Y. Montiel, F. Saidi, F. Hassen, and H. Marref, Opt. Mater. (Amsterdam, Neth.) 24, 303 (2003)

${ }^{31}$ L. F. Bian, D. S. Jiang, P. H. Tan, S. L. Lu, B. Q. Sun, L. H. Li, and J. C. Harmand, Solid State Commun. 132, 707 (2004).

${ }^{32}$ H. Yaguchi, S. Kikuchi, Y. Hijikata, S. Yoshida, D. Aoki, and K. Onabe, Phys. Status Solidi B 228, 273 (2001).

${ }^{33}$ R. Teissier, D. Sicault, J. C. Harmand, G. Ungaro, G. Le Roux, and L. Largeau, J. Appl. Phys. 89, 5473 (2001). 